Document downloaded from:

http://hdl.handle.net/10251/112407

This paper must be cited as:

Pérez-Simbor, S.; Barbi, M.; Garcia-Pardo, C.; Castelló-Palacios, S.; Cardona Marcet, N. (2018). Initial UWB in-body channel characterization using a novel multilayer phantom measurement setup. IEEE. 384-389. doi:10.1109/WCNCW.2018.8369011

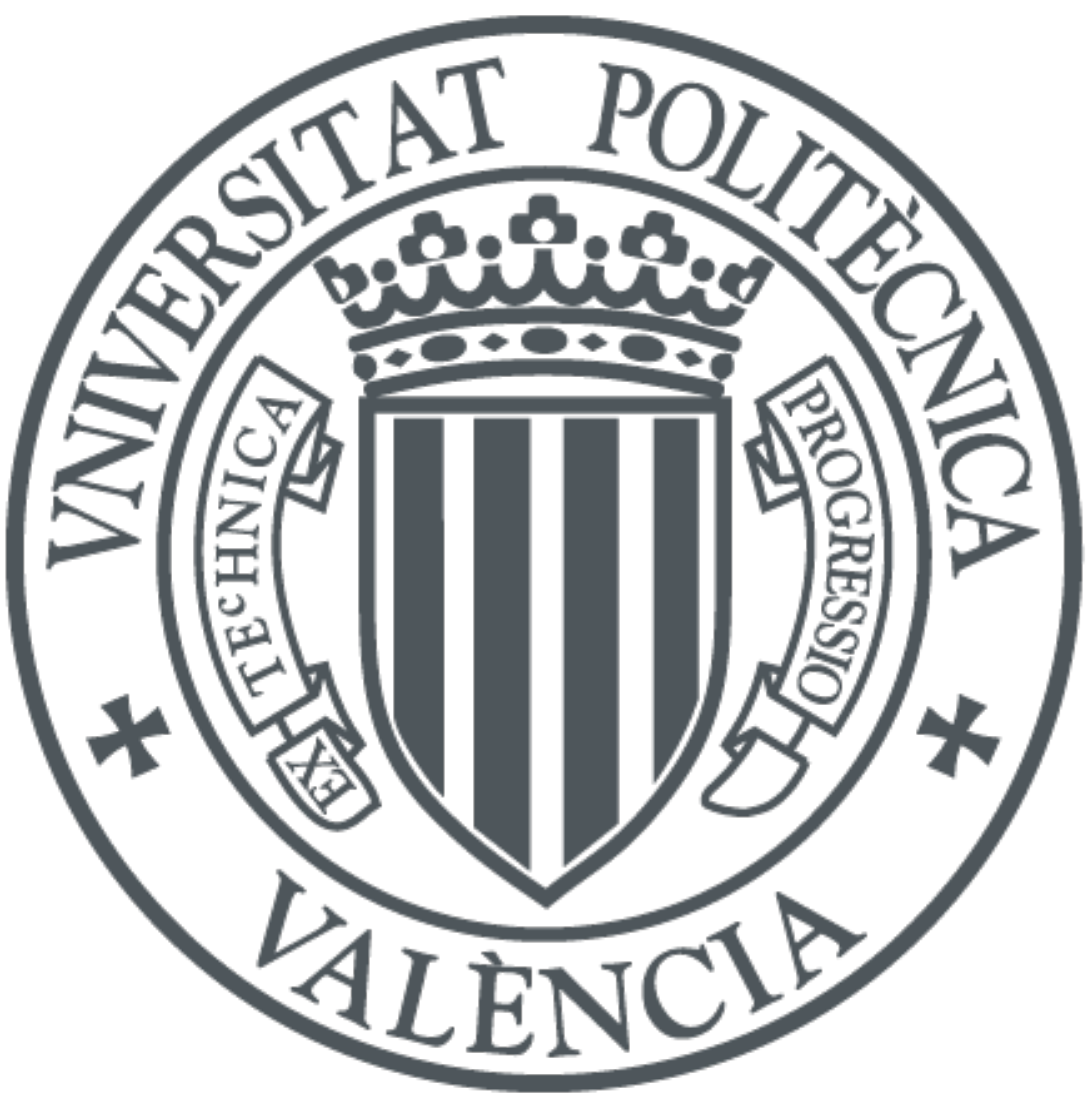

The final publication is available at

http://doi.org/10.1109/WCNCW.2018.8369011

Copyright IEEE

Additional Information 


\title{
Initial UWB In-Body Channel Characterization Using a Novel Multilayer Phantom Measurement Setup
}

\author{
Sofia Perez-Simbor, Martina Barbi, Concepcion Garcia-Pardo, Sergio \\ Castelló-Palacios, Narcís Cardona \\ Institute of Telecommunications and Multimedia Applications (iTEAM) \\ Universitat Politècnica de València (UPV) \\ Valencia, Spain \\ sopresim@iteam.upv.es
}

\begin{abstract}
Wireless Body Area Networks (WBANs) are a promising technology for medical purposes. Currently the WBAN are classified into: implanted (in-), surface (on-) or outside (off-) body communications regarding the location of the devices with reference to the human body. The Ultra Wide-Band (UWB) frequency band is growing as a band of interest for implanted communications because of its high data rate and low power consumption among other benefits. Software simulations, in-vivo measurements and experimental phantom measurements are common methods to properly characterize the propagation channel. Nevertheless, up to now, experimental phantoms measurements presented in the literature show some inconveniences, i.e., the accuracy of the phantoms compared with the real human tissues or the testbed used for the measurements. This paper aims at overcoming these issues using accurate phantoms designed for the purpose of implanted communications in the UWB frequency band. In addition, a multilayer phantom container was developed. This container has capacity for two different phantoms, emulating a heterogeneous propagation medium for in-body measurements. Moreover, a novel setup was built for in-body phantom measurements. As a result, an experimental path loss model is presented from the measurements obtained with phantoms. Besides, software simulations mimicking the experimental setup are performed in order to validate the previous results obtained.
\end{abstract}

Keywords- Ultra Wide-Band (UWB); Wireless Body Area Network (WBAN); phantom measurements; implanted antenna; multilayer measurement setup.

\section{INTRODUCTION}

Wireless Body Area Networks (WBAN) are a promising technology for medical purposes. WBAN are based on the communications around the human body either inside or outside the body. Some medical devices already exist in the market, which use this kind of technology, either from in-body to onbody (IB2OB), on-body to on-body (OB2OB) or on-body to offbody (OB2OFF).

Nowadays, the standard IEEE.802.15.6 [1] regulates the frequency bands that might be used for all the medical applications. The most common frequency bands to use are: Medical Implant Communication Service (MICS), from $402-$ $405 \mathrm{MHz}$, Industrial, Scientific and Medical radio band (ISM) from $2.4-2.4835 \mathrm{GHz}$ and the Ultra Wide-Band frequency band (UWB) from 3.1 to $10.6 \mathrm{GHz}$. However, as described for the current standard [1], for communications from inside the human body (in-body), only the MICS band is considered. This narrow frequency band has a good propagation behavior and small size of the antennas. However, its small bandwidth can lead to low data rate up to $455 \mathrm{kbps}$ [1]. On the contrary, the Ultra WideBand (UWB) frequency band is described in the standard as a band for communications out of the body, either OB2OB or OB2OFF. Nevertheless, UWB frequency band is also growing the attention of the scientific community for in-body communications. The ultra-low power consumption, the high data rate and the small size of the antennas are desired characteristics for such implanted communications [2].

Commonly, the channel characterization is based on software simulations [3]-[5] and experimental measurements, which are classified in: in-vivo [6] and phantom measurements [7]. However, animal experimentation has some ethical issues and high complexity, which reduces the amount of this kind of experiments. Phantom-based measurements are standing out because of their simplicity compared with in-vivo experiments [8], [9]. Phantoms are aqueous solutions that precisely mimic the electromagnetic properties of the human tissues and measurements can be done in laboratory environment. However, current phantoms are mostly narrowband-phantoms, designed for only one single frequency. They are easy to develop, whereas UWB phantoms are difficult to achieve. Recently, some researchers at the Universitat Politècnica de València (UPV) developed a phantom formulation that properly mimic the electromagnetic properties of body tissues given by Gabriel in [10], whose values are considered as a reference for the scientific community. These phantoms achieve a high level of accuracy in the band from 0.5 to $18 \mathrm{GHz}$ [11]. Moreover, they are protected by the patent [12].

Previous researches, present some inaccuracies regarding phantom measurements, as said, the phantoms were not designed for the UWB frequency band, which leads to inaccurate results. Furthermore, measurements considering only one phantom are performed [13], which is not the real in-body scenario because the human body has different tissues. Besides, some measurements setups are not designed for in-body experimentation leading to some imprecisions due to the reflections done in laboratory. 
In this paper, some improvements regarding experimental phantom measurements were achieved. Firstly, the phantoms used are properly designed for the purpose of in-body channel characterization in UWB frequency band. Secondly, a novel multilayer phantom container is developed, achieving two-layer phantom measurements. Moreover, a dedicated setup was developed for in-body phantom experimentation. Finally, experimental results were obtained and compared with software simulations.

This paper is organized as follows: section II describes the measurement setup developed and the methodology used for the experimental measurements in phantom, section III describes some initial results obtained: the channel transfer function, and the experimental path loss models obtained from such measurements. Furthermore, this section presents the software simulations performed to validate the experimental results. And finally section IV summarizes the research and the results presented.

\section{MEASUREMENT SYSTEM AND METHODOLOGY}

\section{A. Measurement system}

Fig. 1 shows the implemented setup used for these experimental phantom measurements. It consists of a Vector Network Analyzer (VNA), a laptop and an anechoic chamber. Inside this anechoic chamber a 3D spatial positioner, a phantom container and a magnetic tracker are placed. The testbed is software controlled by a laptop so that all the elements of the setup are connected to it.

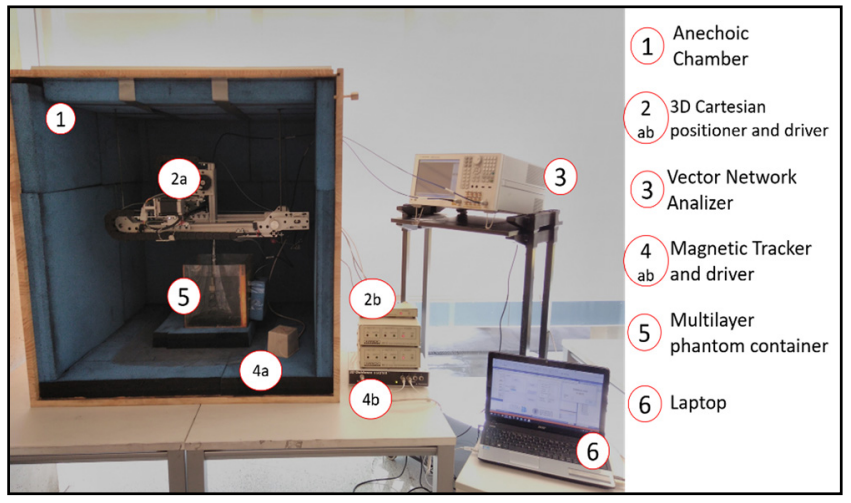

Fig. 1. Full setup

The primary elements of the setup are the anechoic chamber and the elements contained inside Fig. 1. The anechoic chamber is used to minimize the undesired contributions due to the surrounding environment, as in [14]. In Fig. 1, the anechoic chamber opened from one side and the elements contained inside are shown. The chamber has an internal volume of $1 \times 1$ $\times 1 \mathrm{~m}^{3}$ and the structure is built with wood of $22 \mathrm{~mm}$ width. The interior of the chamber is coated with aluminum foil of $13 \mu \mathrm{m}$ width. This coating creates a sort of Faraday Cage for the frequencies under study (UWB), isolating the inside of the cage from the outside. Then, the aluminum foil is re-covered with a flat absorber ( $M V G$-AEMI, $A E L-2.25)$. These absorbers are designed to attenuate at least $20 \mathrm{~dB}$ for frequencies above $1.3 \mathrm{GHz}$.
For in-body scenarios, the separation distance between antennas is in the range of a few centimeters. Therefore, a bad estimation of the antenna location caused by their manual placement, could lead to large relative errors. To overcome this issue, a 3D Cartesian positioner (Arrick Robotics tGlide 3-axis positioner) is placed inside the anechoic chamber (Fig. 1 element 2a). This positioner has a $1 \mathrm{~mm}$ precision along the $X$, $\mathrm{Y}, \mathrm{Z}$ axis and $30 \times 30 \times 10 \mathrm{~cm}^{3}$ movement range. The in-body antenna is attached to the positioner so that it can be accurately moved, enhancing the precision of the measurement. This positioner is connected to a driver, which is located outside the chamber through an electrical connection (Fig. 1, element 2b).

In addition to the accuracy provided by the antenna positioning system, the spatial position of each antenna is given by a 3D magnetic tracker from Ascension Technology Corporation (trakStar with a Mid-Range Transmitter). The mechanism is based on the creation of a magnetic field by the transmitter (Fig. 1, element 4a) inside the anechoic chamber, covering the space under measurement. Then, a magnetic sensor is attached to the center of each antenna. Magnetic transmitter and sensors are then connected to an external driver (Fig. 1, element $4 \mathrm{~b}$ ), where the spatial position of the sensors can be calculated.

The multilayer phantom container (Fig. 1, element 5) is shown in detail in Fig. 2. It is made of polyethylene terephthalate (PET) of $1.5 \mathrm{~mm}$ width and has an overall internal volume of 25 $\times 25 \times 25 \mathrm{~cm}^{3}$ with two layers of $23 \times 25 \times 25$ and $2 \times 25 \times 25$ $\mathrm{cm}^{3}$ for the muscle phantom and the fat phantom respectively. Between layers, there is a divider sheet also made of PET of 1.5 $\mathrm{mm}$.

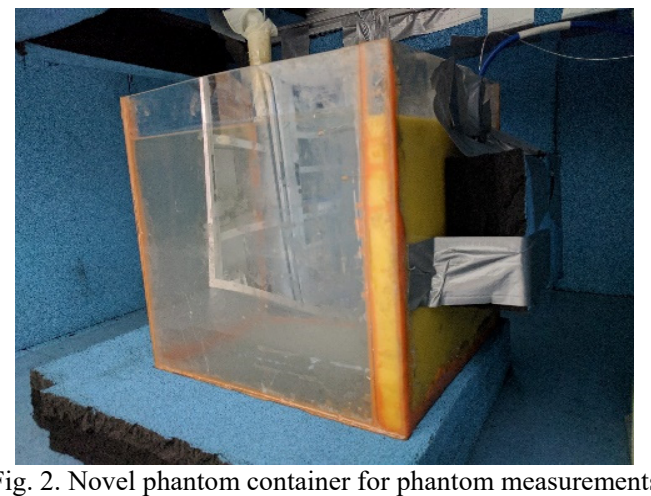
and the on-body antenna covered with absorber

In experimental aqueous phantom-based measurements, a container is filled with liquid phantom. Moreover, the walls of the container are enforced to be as thin as possible in order to have a minimum impact on the propagation through the multilayer phantom.

Considering the scenario of a Wireless Capsule Endoscope (WCE), the main tissues involved are colon, muscle, and fat. Due to the similar permittivity of muscle and colon, only one has been considered for measurements, i.e., muscle tissue, which is the most used in literature for in-body measurements. In addition, as described above, the phantoms created at the UPV accurately mimic human tissues in the entire UWB frequency band. Fig. 3 shows the comparison between the dielectric 
constant and the conductivity of the phantoms with their respective tissues reported in [10], widely used in literature. In Fig. 3 a) the muscle phantom composed with Acetonitrile (54.98\%) and $\mathrm{NaCl}(1.07 \%)$ [11] is shown. Whereas, in Fig. 3 b) The fat phantom used is a simplified version of the original phantom proposed in [15], nonetheless, this version achieves also a good approximation of the electromagnetic properties of the tissues aforementioned in [10]. In this case, the fat phantom is an emulsion of $86 \%$ of oil in water, in which $1 \%$ of TX-100 was used as surfactant.
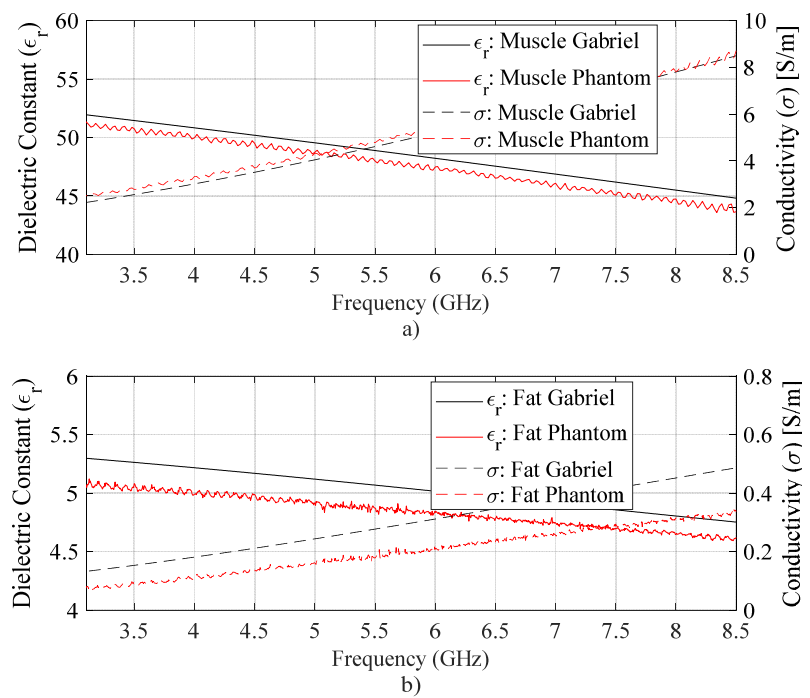

Fig. 3. Phantoms: dielectric constant and conductivity a) Muscle b) Fat.

Finally, the antennas used were already designed for the purpose of body communications in the UWB frequency band. Both are patch antennas with a quasi-omnidirectional radiation pattern and $2.3 \mathrm{~cm} \times 2 \mathrm{~cm}$ and $5 \mathrm{~cm} \times 4.4 \mathrm{~cm}$ length and width dimensions for the in- and on-body antennas respectively [16]. Fig. 4 shows the reflection coefficient of both antennas inside muscle phantom. Further details regarding both antennas can be found in [16] and [17].

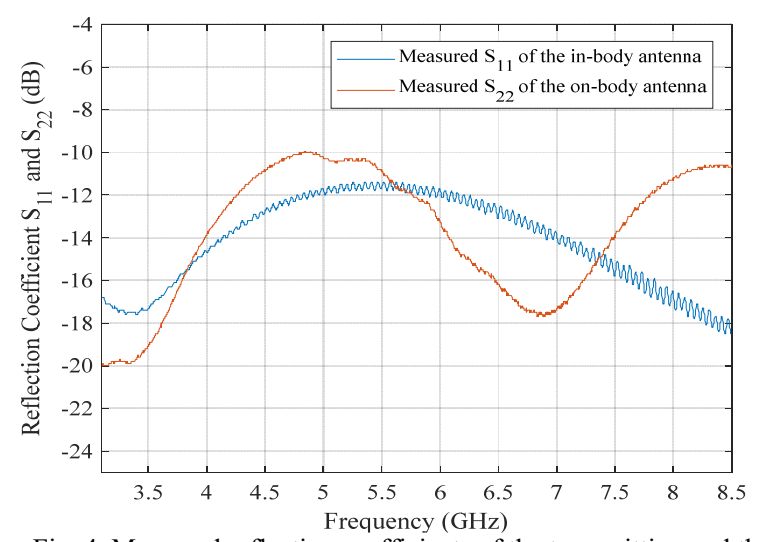

Fig. 4. Measured reflection coefficients of the transmitting and the receiving antennas inside muscle phantom

\section{B. Methodology}

For measurements, the forward transmission coefficient $\left(\mathrm{S}_{21}\right)$ is calculated for different positions of the in-body and on-body antennas. Firstly, the VNA (Agilent ENA E5072A) is calibrated in order to suppress the effect of the coaxial cables and connectors through a full-calibration method using a Rosenberger Calibration Kit RPC - 3.50. The measurement setup parameters were set as given in Table 1.

Five snapshots were taken in order to enhance the Signal-toNoise Ratio (SNR), and then an average value per position is calculated.

TABLE 1

MEASUREMENT SETUP PARAMETERS

\begin{tabular}{cl}
\hline \hline Resolution points & $N=3201$ \\
\hline Frequency band & $f=[3.1,8.5] \mathrm{GHz}$ \\
\hline Resolution frequency & $\Delta f=1.875 \mathrm{MHz}$ \\
\hline Resolution Bandwidth & $f_{i f}=3 \mathrm{kHz}$ \\
\hline Output Power & $P=8 \mathrm{dBm}$ \\
\hline Noise level & $N L=-90 \mathrm{~dB}$ \\
\hline Snapshots per position & $\mathrm{Ns}=5$ \\
\hline \hline
\end{tabular}

The phantom container is then placed inside the chamber and the muscle and fat-phantom were poured in their respective compartments. It should be mentioned that since the phantoms properties vary with temperature, the latter was strictly controlled at $24^{\circ} \mathrm{C}$ by a thermostat.

As mentioned before, the magnetic sensors attached to the antennas provide their XYZ position information. The effect of the sensors over the antennas was tested and it was checked to be negligible. Moreover, in order to avoid inaccuracies, for each snapshot, the position is calculated 100 times. Then, the average value of the distance is taken for each snapshot.

Measurements were done for different positions of the inbody and on-body antennas, concretely in a 3D and 2D grid respectively as shown in Fig. 5

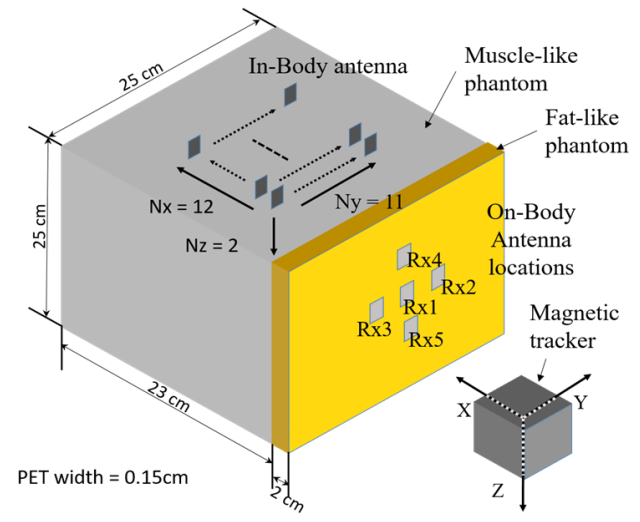

Fig. 5. Measurement Grid Setup [18].

The in-body antenna was attached to the 3D Cartesian positioner, and then submerged in the muscle layer. Moreover, it is recovered with a latex layer to avoid a short between the patch and the phantom. The in-body antenna was moved in steps 
of $\Delta x=\Delta y=\Delta z=1 \mathrm{~cm}$ along $\mathrm{X}, \mathrm{Y}$ and $\mathrm{Z}$ axis with a grid size of $(N x, N y, N z)=(12,11,2)$. Considering the fat layer to be the $\mathrm{YZ}$ plane and the center of the layer the reference position, the $\mathrm{X}$ axis moves closer or farther from the reference plane, the $\mathrm{Y}$ axis moves left or right and the $\mathrm{Z}$ axis moves up and down from the reference position.

The on-body antenna is located in different positions over the fat phantom layer. Moreover, the antenna is covered with the same absorber of the anechoic chamber (Fig. 2) in order to reduce possible small contributions due to reflections from the elements contained in the chamber themselves. In Fig. 5, a cross-shape grid used for the receiving antennas is located over the layer depicted in yellow color, i.e., the fat-like phantom, where each receiver is $2 \mathrm{~cm}$ apart from the central receiver (Rx1).

\section{Measurement AND Simulation Results}

\section{A. Experimental Path Loss Models.}

From the forward transmission coefficient $\mathrm{S}_{21}(\mathrm{f})$ with $\mathrm{N}$ resolutions points (Table 1) obtained from the measurements, the path loss per spatial position was calculated as

$$
P L(d B)=-10 \log _{10}\left(\sum_{i=N} \frac{\left(\left|H\left(f_{i}\right)^{2}\right|\right)}{N}\right)
$$

being $H(f)$ the channel transfer function with $\mathrm{N}$ resolution points, where $H(f)=\left|S_{21}\right| e^{-j \nless S_{21}}$, being $\left|S_{21}\right|$ and $\Varangle S_{21}$ the module and the phase of the transmission coefficient respectively.

In Fig. 6 the channel transfer function is depicted. Each line represents the absolute value of the forward transmission coefficient $\left(\mathrm{S}_{21}\right)$ as a function of the frequency for different distances between antennas. For simplicity, the misalignment between antennas was selected to be negligible, considering always $2 \mathrm{~cm}$ of fat layer between antennas.

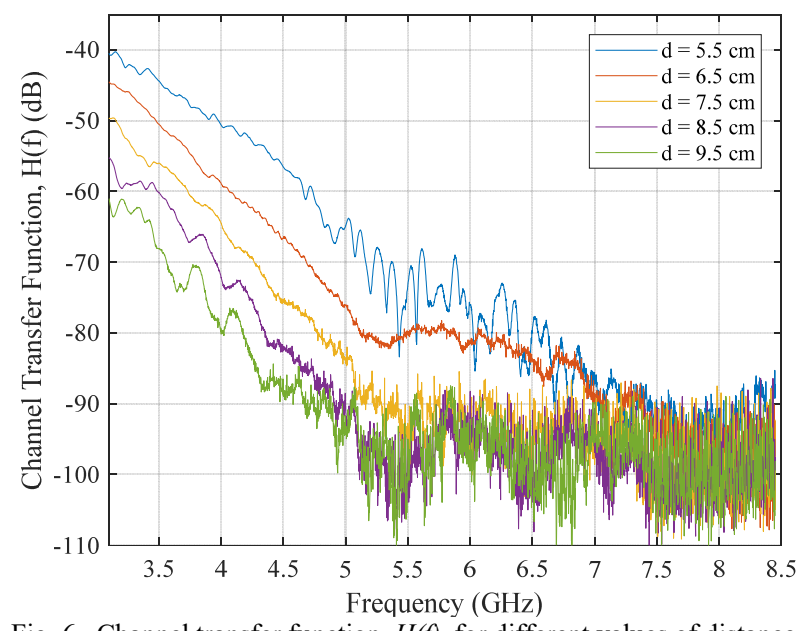

Fig. 6. Channel transfer function, $H(f)$, for different values of distance.

Accordingly to the noise level given in Table 1, all the data values below $-90 \mathrm{~dB}$ are considered to be under the noise level, i.e., they are negligible. Furthermore, from Fig. 6, the tendency of the channel transfer function with the distance is examined. For a distance of $d=5.5 \mathrm{~cm}$, the $H(f)$ has a constant decay until approximately $5.5 \mathrm{GHz}$. For $\mathrm{d}=6.5-9.5 \mathrm{~cm}$ the decay arrives until $f=5.1 \mathrm{GHz}$. These curves show a clear response considering the noise negligible until $f=5.1 \mathrm{GHz}$ and with a power difference of approximately $8 \mathrm{~dB}$ between them. This power decay agrees with the results obtained in [19] where the decay is $7.4 \mathrm{~dB} / \mathrm{cm}$ for homogeneous phantom measurements.

From the measurements obtained, the path loss from $f=3.1$ to $5.1 \mathrm{GHz}$ and $d_{\max }=9.5 \mathrm{~cm}$ is depicted in Fig. 7. As already mentioned, only the measurements with power above the noise level are computed. The path loss obtained can be modeled as a distance-dependent logarithmic function (2), where the shadowing term is given by $N(\mu, \sigma)$, a normal distribution with standard deviation $\sigma=2.307$ and zero mean

$$
P L(d B)=P L_{0}+10 n \log _{10}\left(d / d_{0}\right)+N(\mu, \sigma)
$$

In Table 2, two models of path loss are described, the first considering $d_{0}=1 \mathrm{~cm}$ and the second considering $d_{0}$ as the first distance of all the measurements. Both models are also depicted in Fig. 7, where it is easy to see the overlapping lines corresponding to both PL models.

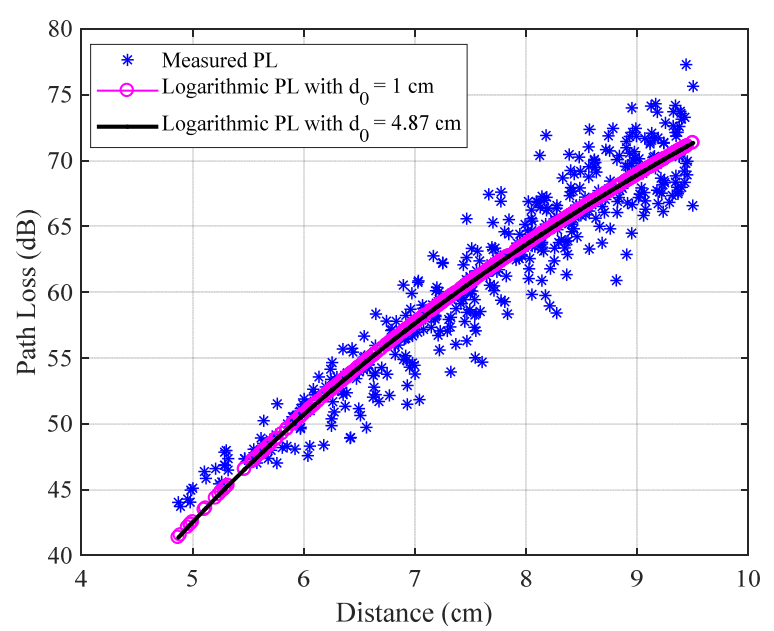

Fig. 7. Experimental Path Loss

TABLE 2. PATH LOSS MODELS FOR MULTILAYER PHANTOM MEASUREMENTS

\begin{tabular}{c|c}
\hline \hline$d_{0}=1 \mathrm{~cm}$ & $d_{0}=4.8717 \mathrm{~cm}$ \\
\hline$P L_{0}=-29.7593 \mathrm{~dB}$ & $P L_{0}=41.3431 \mathrm{~dB}$ \\
\hline$n=10.3395$ & $n=10.3395$ \\
\hline$N(\mu, \sigma)$ & $N(\mu, \sigma)$ \\
$\sigma=2.307$ & $\sigma=2.307$ \\
$\mu=3.1308 \mathrm{e}-14 \approx 0$ & $\mu=5.1605 \mathrm{e}-15 \approx 0$ \\
\hline
\end{tabular}

The first case, with a reference distance of $1 \mathrm{~cm}$, is the most used in the literature. Nevertheless, the reference values are virtual values, because they were not measured in this measurement campaign. On the other hand, the second case is the most realistic one, where the reference values are real measurements obtained, being $d_{0}$ the first distance for which the PL model is valid. 
Moreover, similar studies are present in the literature, in which some similar results are achieved. e.g., in [20], where they performed software simulations for UWB frequency band from 3.4 to $4.8 \mathrm{GHz}$, with reference distance of $5 \mathrm{~cm}$ and $48.6 \mathrm{~dB}$ of $P L_{0}$. In this case, they calculated different PL exponents for different frequency bandwidths.

\section{B. Simulation of the experimental setup}

Measurements were replicated with a commercial software (CST MW Studio ${ }^{\circledR}$ ) for the validation of the results. Nevertheless, an ideal scenario was considered. Fig. 8 shows the software design, which consists in the phantom container, the muscle and fat phantoms and the in- and on-body antennas.

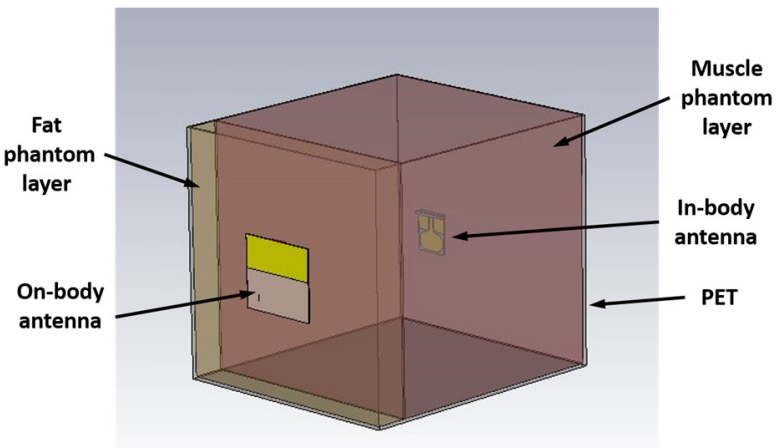

Fig. 8. Measurement Setup Software Design

In this case, both antennas were faced, being both of them aligned with the front plane (fat phantom layer). Therefore, the misalignment between them is considered negligible. In addition, the in-body antenna is placed in the center of the phantom container, whereas the on-body antenna is $3 \mathrm{~mm}$ away from the center of the external side of the fat phantom layer. The only variable in the simulations was the distance between antennas, which ranged from 5.5 to $9.5 \mathrm{~cm}$, being $1 \mathrm{~cm}$ the distance between measurements. As well as in the experimental setup, the in-body and on-body antenna were the transmitting and the receiving antenna respectively. In addition, the forward transmission coefficient $\left(\mathrm{S}_{21}\right)$ was calculated.

It should be mentioned that the computational time and memory required for the software simulations are critical parameters. And for that reason there should be a compromise between the size of the model, the resolution obtained and the time and memory required. In our case, the maximum distance between antennas is $d_{\max }=9.5 \mathrm{~cm}$, allowing the container to be reduced without consequences in the measurements for the desired band and distance. Therefore, the size of the container is set as $17 \times 15 \times 15 \mathrm{~cm}^{3}$ with two layers of $15 \times 15 \times 15$ and $2 \times 15 \times 15 \mathrm{~cm}^{3}$. With this size reduction, the mesh used for the software is higher, i.e., more accurate, but the time and memory resources decrease.

In Fig. 9, the channel transfer function obtained from simulations and the experimental results are depicted. The simulations (dotted lines) and the experimental measurements (continuous lines) are in a good level of agreement, considering the aforementioned PL models valid for the frequency and distance range of 3.1 to $5.1 \mathrm{GHz}$ and $\mathrm{d}_{\max }=9.5 \mathrm{~cm}$. Moreover, in Fig. 9 for a distance of $9.5 \mathrm{~cm}$ the measurements disagree for values below $-90 \mathrm{~dB}$. As said, the noise level of the antennas is $-90 \mathrm{~dB}$. Therefore, the experimental measurements below this threshold are considered noise. As expected, it does not happen with software simulation because since it is an ideal scenario, there is no noise in the system.

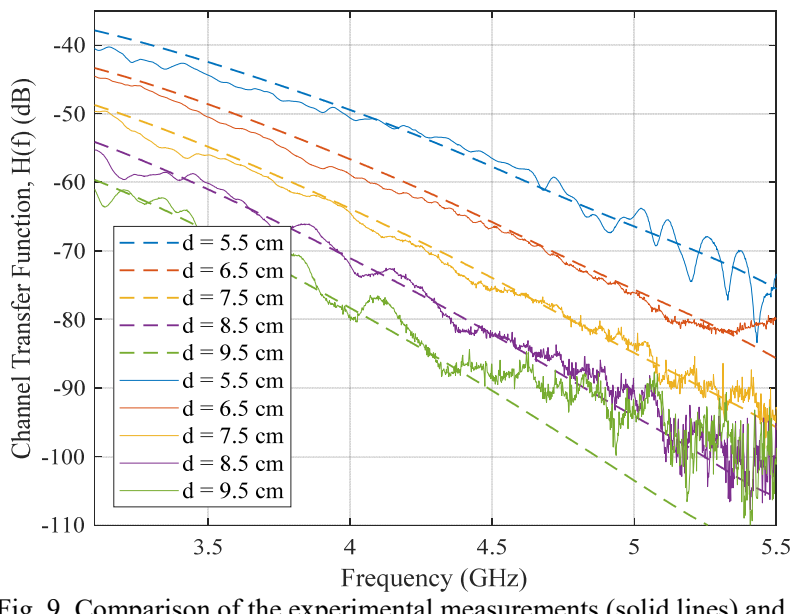

Fig. 9. Comparison of the experimental measurements (solid lines) and software simulations (dotted lines).

In addition, the path loss of the software simulations was calculated. The results show high coincidence with the experimental results, where the simulated PL is coincident with the lowest experimental PL for each given distance. This is, because in software simulations the antenna alignment is always perfect. Therefore, the losses due to the misalignment are negligible.

\section{CONCLUSIONS}

In this work, an experimental in-body measurement campaign in the UWB frequency band was performed. For this purpose, a multi-layer measurement setup was developed for inbody phantom measurements. This measurement setup, among others, has the following elements: a small anechoic chamber, a Vector Network Analyzer, a 3D Cartesian positioner, a magnetic tracker and a two-layer phantom container, filled with muscle and fat phantom. Then an experimental path loss (PL) model was obtained for the measurements. This PL model is defined for the UWB frequency band, concretely from $f=3.1$ to $5.1 \mathrm{GHz}$ and for a distance range of 4.87 to $9.5 \mathrm{~cm}$. Finally, the experimental results were validated with software simulations. From simulations, the channel transfer function for different distances were compared with the equivalent result in experimental measurements, showing both measurements a good agreement between them.

As a future research, in-vivo measurements have to be done to double check the experimental measurements in phantom. Moreover, regarding future phantom measurements, the enhancing of the phantom container is a key factor. Measurements with more complex phantom container shapes as well as multilayers containers, which are closer to the real human body environment are required. 


\section{ACKNOWLEDGMENT}

This work was supported by the European Union's H2020:MSCA:ITN program for the "Wireless In-body Environment Communication- WiBEC" project under the grant agreement no. 675353. This work was also funded by the Programa de Ayudas de Investigación y Desarrollo (PAID-0116) from Universitat Politècnica de València and by the Ministerio de Economía y Competitividad, Spain (TEC201460258-C2-1-R), by the European FEDER funds.

\section{REFERENCES}

[1] IEEE Standards Association, IEEE Standard for Local and metropolitan area networks - Part 15.6: Wireless Body Area Networks, February. 2012.

[2] A. Ghildiyal, K. Amara, R. D. Molin, B. Godara, A. Amara, and R. K. Shevgaonkar, "UWB for in-body medical implants: A viable option," in 2010 IEEE International Conference on Ultra-Wideband, 2010, pp. 1-4.

[3] A. Khaleghi, R. Chávez-Santiago, and I. Balasingham, "Ultrawideband statistical propagation channel model for implant sensors in the human chest," IET Microwaves, Antennas Propag., vol. 5, no. 15 , p. $1805,2011$.

[4] H. Bahrami, S. A. Mirbozorgi, L. A. Rusch, and B. Gosselin, "Biological Channel Modeling and Implantable UWB Antenna Design for Neural Recording Systems," IEEE Trans. Biomed. Eng., vol. 62, no. 1, pp. 88-98, 2015.

[5] A. Khaleghi, R. Chavez-Santiago, and I. Balasingham, "An improved ultra wideband channel model including the frequency-dependent attenuation for in-body communications," in Proceedings of the Annual International Conference of the IEEE Engineering in Medicine and Biology Society, EMBC, 2012, pp. 1631-1634.

[6] C. Garcia-Pardo, R. Chavez-Santiago, N. Cardona, and I. Balasingham, "Experimental UWB frequency analysis for implant communications," in Proceedings of the Annual International Conference of the IEEE Engineering in Medicine and Biology Society, EMBS, 2015, vol. 2015-Novem, pp. 5457-5460.

[7] C. Garcia-pardo, C. Andreu, A. Fornes-leal, S. Castelló-palacios, S. Perez-Simbor, M. Barbi, A. Vallés-lluch, and N. Cardona, "UWB Propagation Environment for Medical In-Body Sensor Networks," IEEE Antennas and Propagation Magazine, Accepted, 2018.

[8] R. Chavez-Santiago, C. Garcia-Pardo, A. Fornes-Leal, A. VallesLluch, I. Balasingham, and N. Cardona, "Ultra wideband propagation for future in-body sensor networks," IEEE Int. Symp. Pers. Indoor Mob. Radio Commun. PIMRC, vol. 2015-June, pp. 2160-2163, 2015.

[9] H. Yamamoto, J. Zhou, and T. Kobayashi, "Ultra wideband electromagnetic phantoms for antennas and propagation studies,"
IEICE Trans. Fundam. Electron. Commun. Comput. Sci., vol. E91A, no. 11, pp. 3173-3182, 2008.

[10] C. Gabriel, "Compilation of the Dielectric Properties of Body Tissues at RF and Microwave Frequencies.," Environ. Heal., June, p. 21, 1996.

[11] S. Castelló-Palacios, C. Garcia-Pardo, A. Fornes-Leal, N. Cardona, and A. Vallés-Lluch, "Tailor-Made Tissue Phantoms Based on Acetonitrile Solutions for Microwave Applications up to $18 \mathrm{GHz}$," IEEE Trans. Microw. Theory Tech., vol. 64, no. 11, pp. 3987-3994, 2016.

[12] N. Cardona, S. Castelló Palacios, A. Fornés Leal, C. García Pardo, and A. Vallés Lluch, "Synthetic Model of Biological Tissues for Evaluating the Wireless Transmission of Electromagnetic Waves," Patent WO/2017/109252, 2017.

[13] C. Andreu, S. Castello-Palacios, C. Garcia-Pardo, A. Fornes-Leal, A Valles-Lluch, and N. Cardona, "Spatial In-Body Channel Characterization Using an Accurate UWB Phantom," IEEE Trans. Microw. Theory Tech., 2016.

[14] T. Kumpuniemi, T. Tuovinen, M. Hämäläinen, K. Y. Yazdandoost, R. Vuohtoniemi, and J. Iinatti, "Measurement-based on-body path loss modelling for UWB WBAN communications," Int. Symp. Med. Inf. Commun. Technol. ISMICT, pp. 233-237, 2013.

[15] M. Lazebnik, E. L. Madsen, G. R. Frank, and S. C. Hagness, “Tissuemimicking phantom materials for narrowband and ultrawideband microwave applications.," Phys. Med. Biol., vol. 50, no. 18, pp. 4245-58, Sep. 2005.

[16] C. Andreu, C. Garcia-Pardo, A. Fornes-Leal, M. Cabedo-Fabrés, and N. Cardona, "UWB In-Body Channel Performance by Using a Direct Antenna Designing Procedure," in 11th European Conference on Antennas and Propagation (EUCAP), 2017, p. 5.

[17] C. Tarin, P. Marti, L. Traver, N. Cardona, J. A. Diaz, and E. Antonino, "UWB channel measurements for hand-portable devices: A comparative study," IEEE Int. Symp. Pers. Indoor Mob. Radio Commun. PIMRC, 2007.

[18] M. Barbi, S. Perez-Simbor, C. Garcia-Pardo, S. Castelló-Palacios, C. Andreu, A. Fornes-Leal, and N. Cardona, "Localization for Capsule Endoscopy at UWB Frequencies using an Experimental Multilayer Phantom," in IEEE Wireless Communications and Networking Conference, WCNC, 2018, Submitted.

[19] C. Garcia-Pardo, A. Fornes-Leal, N. Cardona, R. Chavez-Santiago, J. Bergsland, I. Balasingham, S. Brovoll, O. Aardal, S.-E. Hamran, and R. Palomar, "Experimental ultra wideband path loss models for implant communications," 2016 IEEE 27th Annu. Int. Symp. Pers. Indoor, Mob. Radio Commun., pp. 1-6, 2016.

[20] J. Wang, Y. Shimizu, D. Anzai, and T. Furukawa, "Performance improvement by transmit diversity technique for implant ultrawideband communication," IET Microwaves, Antennas Propag., vol. 10, no. 10, pp. 1106-1112, 2016. 